\title{
THE QUASI-HADAMARD PRODUCTS OF CERTAIN $p$-VALENT FUNCTIONS WITH NEGATIVE COEFFICIENTS
}

\author{
Mohamed Kamal Aouf
}

\begin{abstract}
The object of the present paper is to show quasi-Hadamard products of certain $p$-valent functions with negative coefficients in the open unit disc. Our results are the generalizations of the corresponding results due to Yaguchi et al. [10], Aouf and Darwish [3], Lee et al. [5] and Sekine and Owa [9].
\end{abstract}

\section{Introduction}

Let $A_{p}(n)$ be the class of functions of the form :

$$
f(z)=z^{p}-\sum_{k=p+n}^{\infty} a_{k} z^{k}\left(a_{k} \geq 0 ; p, n \in N=\{1,2, \ldots\}\right)
$$

which are analytic and $p$-valent in the unit disc $U=\{z:|z|<1\}$. A function $f(z) \in A_{p}(n)$ is said to be a member of the class $P_{p}^{*}(n, \alpha, \beta)$ if it satisfies

$$
\left|\frac{\frac{f^{\prime}(z)}{z^{p-1}}-p}{\frac{f^{\prime}(z)}{z^{p-1}}+p-2 \alpha}\right|<\beta \quad(z \in U)
$$

for some $\alpha(0 \leq \alpha<p)$ and $\beta(0<\beta \leq 1)$. The class $P_{p}^{*}(n, \alpha, \beta)$ was studied by Aouf $[1,2]$.

We note that :

(i) For $\beta=1$, the class $P_{p}^{*}(n, \alpha, 1)=P_{p}^{*}(n, \alpha)=\left\{f(z) \in A_{p}(n)\right.$ : $\left.\operatorname{Re}\left\{\frac{f^{\prime}(z)}{z^{p-1}}\right\}>\alpha(z \in U), 0 \leq \alpha<p\right\}$ was studied by Yaguchi et al. [10] and Owa and Aouf [7];

(ii) For $\beta=n=1$, the class $P_{p}^{*}(1, \alpha, 1)=P_{p}^{*}(\alpha)=\left\{f(z) \in A_{p}\right.$ : $\left.\operatorname{Re}\left\{\frac{f^{\prime}(z)}{z^{p-1}}\right\}>\alpha(z \in U), 0 \leq \alpha<p\right\}$ was studied by Lee et al. [5], Aouf and Darwish [4] and Aouf [3];

Received December 14, 2005; Revised June 1, 2007. 2000 Mathematics Subject Classification. 30C45. ts.

Key words and phrases. analytic, $p$-valent, quasi-Hadamard product, negative coefficien- 
(iii) For $\beta=p=1$, the class $P_{1}^{*}(n, \alpha, 1)=\widetilde{C}(\alpha, n)=\left\{f(z) \in A_{1}(n)\right.$ : $\left.\operatorname{Re}\left\{f^{\prime}(z)\right\}>\alpha(z \in U), 0 \leq \alpha<1\right\}$ was studied by Sekine and Owa [9];

(iv) For $\beta=p=n=1$, the class $P_{1}^{*}(1, \alpha, 1)=\widetilde{C}(\alpha, 1)$ was studied by Sarangi and Urelagaddi [8] and Owa [6].

For functions $f_{j}(z) \in A_{p}(n)$ defined by

$$
f_{j}(z)=z^{p}-\sum_{k=p+n}^{\infty} a_{k, j} z^{k}\left(a_{k, j} \geq 0 ; j \in N\right),
$$

we denote by $\left(f_{1} * f_{2}\right)(z)$ the quasi-Hadamard product of functions $f_{1}(z)$ and $f_{2}(z)$, that is,

$$
\left(f_{1} * f_{2}\right)(z)=z^{p}-\sum_{k=p+n}^{\infty} a_{k, 1} a_{k, 2} z^{k} .
$$

For $\beta=1$ Yaguchi et al. [10] proved the following results :

Theorem A. If $f_{j}(z) \in P_{p}^{*}\left(n, \alpha_{j}, 1\right)=P_{p}^{*}\left(n, \alpha_{j}\right)(j=1,2)$, then $\left(f_{1} * f_{2}\right)(z) \in$ $P_{p}^{*}(n, \gamma)$, where

$$
\gamma=p-\frac{\prod_{j=1}^{2}\left(p-\alpha_{j}\right)}{p+n}
$$

The result is sharp.

Theorem B. If $f_{j}(z) \in P_{p}^{*}\left(n, \alpha_{j}\right)(j=1,2)$, then the function

$$
h(z)=z^{p}-\sum_{k=p+n}^{\infty}\left(a_{k, 1}^{2}+a_{k, 2}^{2}\right) z^{k}
$$

is in the class $P_{p}^{*}(n, \gamma)$, where

$$
\gamma=p-\frac{2\left(p-\alpha_{0}\right)^{2}}{p+n}\left(\alpha_{0}=\min \left\{\alpha_{1}, \alpha_{2}\right\}\right) .
$$

The result is sharp.

For $\beta=n=1$, Lee et al. [5] have shown that :

Theorem C. If $f_{j}(z) \in P_{p}^{*}(1, \alpha, 1)=P_{p}^{*}(\alpha)(j=1,2)$, then $\left(f_{1} * f_{2}\right)(z) \in$ $P_{p}^{*}(\gamma)$, where

$$
\gamma=p-\frac{(p-\alpha)^{2}}{p+1}
$$

The result is sharp. 
THE QUASI-HADAMARD PRODUCTS OF CERTAIN p-VALENT FUNCTIONS 599

Also for $\beta=n=1$, Aouf and Darwish [3] have proved the following results:

Theorem D. If $f_{j}(z) \in P_{p}^{*}\left(\alpha_{j}\right)(j=1,2)$, then $\left(f_{1} * f_{2}\right)(z) \in P_{p}^{*}(\gamma)$, where

$$
\gamma=p-\frac{\prod_{j=1}^{2}\left(p-\alpha_{j}\right)}{p+1} .
$$

The result is sharp.

Theorem E. If $f_{j}(z) \in P_{p}^{*}(\alpha)(j=1,2,3)$, then $\left(f_{1} * f_{2} * f_{3}\right)(z) \in P_{p}^{*}(\gamma)$, where

$$
\gamma=p-\frac{(p-\alpha)^{3}}{(p+1)^{2}}
$$

The result is sharp.

Theorem F. If $f_{j}(z) \in P_{p}^{*}(\alpha)(j=1,2)$, then the function

$$
h(z)=z^{p}-\sum_{k=p+1}^{\infty}\left(a_{k, 1}^{2}+a_{k, 2}^{2}\right) z^{k}
$$

is in the class $P_{p}^{*}(\gamma)$, where

$$
\gamma=p-\frac{2(p-\alpha)^{2}}{p+1}
$$

The result is sharp.

Further for $\beta=p=1$, Sekine and Owa [9] proved the following results :

Theorem G. If $f_{j}(z) \in P_{1}^{*}(n, \alpha, 1)=\widetilde{C}(\alpha, n)(j=1,2)$, then $\left(f_{1} * f_{2}\right)(z) \in$ $\widetilde{C}(n, \gamma)$, where

$$
\gamma=1-\frac{(1-\alpha)^{2}}{n+1}
$$

The result is sharp.

Theorem H. If $f_{j}(z) \in \widetilde{C}(\alpha, n)(j=1,2)$, then the function

$$
g(z)=z-\sum_{k=n+1}^{\infty}\left(a_{k, 1}^{2}+a_{k, 2}^{2}\right) z^{k}
$$

is in the class $\widetilde{C}(\gamma, n)$, where

$$
\gamma=1-\frac{2(1-\alpha)^{2}}{n+1} .
$$

The result is sharp. 
In the present paper, we prove some interesting generalizations of the theorems given by Yaguchi et al. [10], Aouf and Darwish [3], Lee et al. [5] and Sekine and Owa [9].

\section{Quasi-Hadamard products}

To prove our main results of quasi-Hadamard products, we need the following lemma given by Aouf ([1] and [2]).

Lemma 1. A function $f(z) \in A_{n}(p)$ is in the class $P_{p}^{*}(n, \alpha, \beta)$ if and only if

$$
\sum_{k=p+n}^{\infty}(1+\beta) k a_{k} \leq 2 \beta(p-\alpha) .
$$

Applying the above lemma, we derive :

Theorem 1. If $f_{j}(z) \in P_{p}^{*}\left(n, \alpha_{j}, \beta\right)(j=1,2, \ldots, m)$, then $\left(f_{1} * f_{2} * \cdots *\right.$ $\left.f_{m}\right)(z) \in P_{p}^{*}(n, \gamma, \beta)$, where

$$
\gamma=p-\frac{\prod_{j=1}^{m} 2 \beta\left(p-\alpha_{j}\right)}{2 \beta[(1+\beta)(p+n)]^{m-1}} .
$$

The result is sharp for the functions

$$
f_{j}(z)=z^{p}-\frac{2 \beta\left(p-\alpha_{j}\right)}{(1+\beta)(p+n)} z^{p+n}(j=1,2, \ldots, m) .
$$

Proof. For $m=1$, we see that $\gamma=\alpha_{1}$. For $m=2$, Lemma 1 gives

$$
\sum_{k=p+n}^{\infty} \frac{k(1+\beta)}{2 \beta\left(p-\alpha_{j}\right)} a_{k, j} \leq 1 \quad(j=1,2) .
$$

This gives that

$$
\sum_{k=p+n}^{\infty} \frac{(1+\beta) k}{\sqrt{\prod_{j=1}^{2} 2 \beta\left(p-\alpha_{j}\right)}} \sqrt{a_{k, 1} a_{k, 2}} \leq 1 .
$$

To prove the case when $m=2$, we have to find the largest $\gamma$ such that

$$
\sum_{k=p+n}^{\infty} \frac{(1+\beta) k}{2 \beta(p-\gamma)} a_{k, 1} a_{k, 2} \leq 1,
$$

or such that

$$
\frac{\sqrt{a_{k, 1} a_{k, 2}}}{2 \beta(p-\gamma)} \leq \frac{1}{\sqrt{\prod_{j=1}^{2} 2 \beta\left(p-\alpha_{j}\right)}}(k \geq p+n)
$$


Further, by using (2.5), we need to find the largest $\gamma$ such that

$$
\frac{1}{2 \beta(p-\gamma)} \leq \frac{(1+\beta) k}{\prod_{j=1}^{2} 2 \beta\left(p-\alpha_{j}\right)}(k \geq p+n) .
$$

It follows from (2.8) that

$$
\gamma \leq p-\frac{\prod_{j=1}^{2} 2 \beta\left(p-\alpha_{j}\right)}{2 \beta(1+\beta) k}(k \geq p+n) .
$$

Defining the function $\varphi(k)$ by

$$
\varphi(k)=p-\frac{\prod_{j=1}^{2} 2 \beta\left(p-\alpha_{j}\right)}{2 \beta(1+\beta) k},
$$

we see that $\varphi^{\prime}(k) \geq 0$ for $k \geq p+n$. This implies that

$$
\gamma \leq \varphi(p+n)=p-\frac{\prod_{j=1}^{2} 2 \beta\left(p-\alpha_{j}\right)}{2 \beta(1+\beta)(p+n)} .
$$

Therefore, the result is true for $m=2$.

Suppose that the result is true for any positive integer $m$. Then we have $\left(f_{1} * f_{2} * \cdots * f_{m} * f_{m+1}\right)(z) \in P_{p}^{*}(n, \lambda, \beta)$, where

$$
\lambda=p-\frac{2 \beta(p-\gamma) 2 \beta\left(p-\alpha_{m+1}\right)}{2 \beta(1+\beta)(p+n)},
$$

where $\gamma$ is given by (2.2). After a simple calculation, we have

$$
\lambda=p-\frac{\prod_{j=1}^{m+1} 2 \beta\left(p-\alpha_{j}\right)}{2 \beta[(1+\beta)(p+n)]^{m}} .
$$

Thus, the result is true for $m+1$. Therefore, by using the mathematical induction, we conclude that the result is true for any positive integer $m$.

Finally, taking the functions $f_{j}(z)$ defined by $(2.3)$, we have

$$
\left(f_{1} * f_{2} * \cdots * f_{m}\right)(z)=z^{p}-\left\{\prod_{j=1}^{m} \frac{2 \beta\left(p-\alpha_{j}\right)}{(1+\beta)(p+n)}\right\} z^{p+n}=z^{p}-A_{p+n} z^{p+n},
$$

which shows that

$$
\sum_{k=p+n}^{\infty}\left[\frac{(1+\beta) k}{2 \beta(p-\gamma)}\right] A_{k}
$$




$$
=\frac{(1+\beta)(p+n)}{2 \beta(p-\gamma)}\left\{\prod_{j=1}^{m} \frac{2 \beta\left(p-\alpha_{j}\right)}{(1+\beta)(p+n)}\right\}=1 .
$$

Consequently, the result is sharp.

Putting $\alpha_{j}=\alpha(j=1,2, \ldots, m)$ in Theorem 1 , we have :

Corollary 1. If $f_{j}(z) \in P_{p}^{*}(n, \alpha, \beta)(j=1,2, \ldots, m)$, then $\left(f_{1} * f_{2} * \cdots * f_{m}\right)(z) \in$ $P_{p}^{*}(n, \gamma, \beta)$, where

$$
\gamma=p-\frac{[2 \beta(p-\alpha)]^{m}}{2 \beta[(1+\beta)(p+n)]^{m-1}} .
$$

The result is sharp for the functions

$$
f_{j}(z)=z^{p}-\frac{2 \beta(p-\alpha)}{(1+\beta)(p+n)} z^{p+n}(j=1,2, \ldots, m) .
$$

Putting $\beta=1$ in Theorem 1, we have :

Corollary 2. If $f_{j}(z) \in P_{n}^{*}\left(n, \alpha_{j}, 1\right)=P_{n}^{*}\left(n, \alpha_{j}\right)(j=1,2, \ldots, m)$, then $\left(f_{1} *\right.$ $\left.f_{2} * \cdots * f_{m}\right)(z) \in P_{p}^{*}(n, \gamma)$, where

$$
\gamma=p-\frac{\prod_{j=1}^{m}\left(p-\alpha_{j}\right)}{(p+n)^{m-1}} .
$$

The result is sharp for the functions

$$
f_{j}(z)=z^{p}-\frac{p-\alpha_{j}}{p+n} z^{p+n}(j=1,2, \ldots, m) .
$$

Putting $n=1$ in Corollary 1, we have :

Corollary 3. If $f_{j}(z) \in P_{p}^{*}(1, \alpha, \beta)=P_{p}^{*}(\alpha, \beta)(j=1,2, \ldots, m)$, then $\left(f_{1} * f_{2} *\right.$ $\left.\cdots * f_{m}\right)(z) \in P_{p}^{*}(\gamma, \beta)$, where

$$
\gamma=p-\frac{[2 \beta(p-\alpha)]^{m}}{2 \beta[(1+\beta)(p+1)]^{m-1}} .
$$

The result is sharp for the functions

$$
f_{j}(z)=z^{p}-\frac{2 \beta(p-\alpha)}{(1+\beta)(p+1)} z^{p+1} \quad(j=1,2, \ldots, m) .
$$

Putting $\beta=n=1$ in Corollary 1 , we have : 
Corollary 4. If $f_{j}(z) \in P_{p}^{*}(1, \alpha, 1)=P_{p}^{*}(\alpha)(j=1,2, \ldots, m)$, then $\left(f_{1} * f_{2} *\right.$ $\left.\cdots * f_{m}\right)(z) \in P_{p}^{*}(\gamma)$, where

$$
\gamma=p-\frac{(p-\alpha)^{m}}{(p+1)^{m-1}}
$$

The result is sharp for the functions

$$
f_{j}(z)=z^{p}-\frac{p-\alpha}{p+1} z^{p+1} \quad(j=1,2, \ldots, m) .
$$

Putting $\beta=p=1$ in Corollary 1 , we have :

Corollary 5. If $f_{j}(z) \in P_{1}^{*}(n, \alpha, 1)=\widetilde{C}(n, \alpha)(j=1,2, \ldots, m)$, then $\left(f_{1} * f_{2} *\right.$ $\left.\cdots * f_{m}\right)(z) \in \widetilde{C}(n, \gamma)$, where

$$
\gamma=1-\frac{(1-\alpha)^{m}}{(1+n)^{m-1}}
$$

The result is sharp for the functions

$$
f_{j}(z)=z-\frac{1-\alpha}{1+n} z^{1+n} \quad(j=1,2, \ldots, m) .
$$

Remark 1. (i) Corollary 4 (when $\beta=n=1$ ) is the generalization of Theorem E given by Aouf and Darwish [3];

(ii) Corollary 2 is the generalization of Theorem A given by Yaguchi et al. [10]. Also Corollary 2 (when $n=1$ ) is the generalization of Theorem D given by Aouf and Darwish [3];

(iii) Corollary 4 is the generalization of Theorem $\mathrm{C}$ given by Lee et al. [5];

(iv) Corollary 5 is the generalization of Theorem $G$ given by Sekine and Owa [9].

Theorem 2. If $f_{j}(z) \in P_{p}^{*}\left(n, \alpha_{j}, \beta\right)(j=1,2, \ldots, m)$ and

$$
h(z)=z^{p}-\sum_{k=p+n}^{\infty}\left(\sum_{j=1}^{m} a_{k, j}^{2}\right) z^{k},
$$

then $h(z) \in P_{p}^{*}(n, \gamma, \beta)$, where

$$
\gamma=p-\frac{m\left[2 \beta\left(p-\alpha_{0}\right)\right]^{2}}{2 \beta(1+\beta)(p+n)}\left(\alpha_{0}=\min \left\{\alpha_{1}, \alpha_{2}, \ldots, \alpha_{m}\right\}\right) .
$$

The result is sharp for the functions $f_{j}(z)$ given by (2.3).

Proof. Since Lemma 1 gives

$$
\sum_{k=p+n}^{\infty}\left\{\frac{(1+\beta) k}{2 \beta\left(p-\alpha_{j}\right)}\right\}^{2} a_{k, j}^{2} \leq\left\{\sum_{k=p+n}^{\infty} \frac{(1+\beta) k}{2 \beta\left(p-\alpha_{j}\right)} a_{k, j}\right\}^{2} \leq 1
$$


for $j=1,2, \ldots, m$, we have

$$
\sum_{k=p+n}^{\infty} \frac{1}{m}\left\{\frac{(1+\beta) k}{2 \beta\left(p-\alpha_{j}\right)}\right\}^{2}\left(\sum_{j=1}^{m} a_{k, j}^{2}\right) \leq 1 .
$$

Note that we have to find the largest $\gamma$ such that

$$
\sum_{k=p+n}^{\infty}\left\{\frac{(1+\beta) k}{2 \beta(p-\gamma)}\right\}\left(\sum_{j=1}^{m} a_{k, j}^{2}\right) \leq 1 .
$$

This implies that

$$
\gamma \leq p-\frac{m\left[2 \beta\left(p-\alpha_{0}\right)\right]^{2}}{2 \beta(1+\beta) k} \quad(k \geq p+n)
$$

that is, that

$$
\gamma \leq p-\frac{m\left[2 \beta\left(p-\alpha_{0}\right)\right]^{2}}{2 \beta(1+\beta)(p+n)},
$$

which completes the proof of Theorem 2 .

Putting $\alpha_{j}=\alpha(j=1,2, \ldots, m)$ in Theorem 2, we have :

Corollary 6. If $f_{j}(z) \in P_{p}^{*}(n, \alpha, \beta)(j=1,2, \ldots, m)$ and $h(z)$ is defined by (2.26), then $h(z) \in P_{p}^{*}(n, \gamma, \beta)$, where

$$
\gamma=p-\frac{m[2 \beta(p-\alpha)]^{2}}{2 \beta(1+\beta)(p+n)} .
$$

The result is sharp for the functions $f_{j}(z)$ defined by $(2.17)$.

Putting $\beta=1$ in Theorem 2, we have :

Corollary 7. If $f_{j}(z) \in P_{p}^{*}\left(n, \alpha_{j}\right)(j=1,2, \ldots, m)$ and $h(z)$ is defined by (2.26), then $h(z) \in P_{p}^{*}(n, \gamma)$, where

$$
\gamma=p-\frac{m\left(p-\alpha_{0}\right)^{2}}{p+n}\left(\alpha_{0}=\min \left\{\alpha_{1}, \alpha_{2}, \ldots, \alpha_{m}\right\}\right) .
$$

The result is sharp for the functions $f_{j}(z)$ defined by $(2.19)$.

Putting $\beta=1$ in Corollary 6, we have :

Corollary 8. If $f_{j}(z) \in P_{p}^{*}(n, \alpha)(j=1,2, \ldots, m)$ and $h(z)$ is defined by (2.26), then $h(z) \in P_{p}^{*}(n, \gamma)$, where

$$
\gamma=p-\frac{m(p-\alpha)^{2}}{p+n} .
$$


The result is sharp for the functions $f_{j}(z)$ defined by

$$
f_{j}(z)=z^{p}-\frac{p-\alpha}{p+n} z^{p+n}(j=1,2, \ldots, m) .
$$

Putting $n=1$ in Corollary 6 , we have :

Corollary 9. If $f_{j}(z) \in P_{p}^{*}(\alpha, \beta)(j=1,2, \ldots, m)$ and $h(z)$ is defined by $(2.26)$ with $n=1$, then $h(z) \in P_{p}^{*}(\gamma, \beta)$, where

$$
\gamma=p-\frac{m[2 \beta(p-\alpha)]^{2}}{2 \beta(1+\beta)(p+1)} .
$$

The result is sharp for the functions $f_{j}(z)$ defined by $(2.21)$.

Putting $\beta=n=1$ in Corollary 6 , we have :

Corollary 10. If $f_{j}(z) \in P_{p}^{*}(\alpha)(j=1,2, \ldots, m)$ and $h(z)$ is defined by (2.26) with $n=1$, then $h(z) \in P_{p}^{*}(\gamma)$, where

$$
\gamma=p-\frac{m(p-\alpha)^{2}}{p+1} .
$$

The result is sharp for the functions $f_{j}(z)$ defined by (2.23).

Putting $\beta=p=1$ in Corollary 6 , we have :

Corollary 11. If $f_{j}(z) \in \widetilde{C}(n, \alpha)(j=1,2, \ldots, m)$ and $h(z)$ is defined by (2.26) with $p=1$, then $h(z) \in \widetilde{C}(n, \gamma)$, where

$$
\gamma=1-\frac{m(1-\alpha)^{2}}{1+n} .
$$

The result is sharp for the functions $f_{j}(z)$ defined by (2.25).

Remark 2. (i) Corollary 7 is the generalization of Theorem B given by Yaguchi et al. [10];

(ii) Corollary 10 is the generalization of Theorem $\mathrm{F}$ given by Aouf and Darwish [3];

(iii) Corollary 11 is the generalization of Theorem $\mathrm{H}$ given by Sekine and Owa [9].

Acknowledgement. The author would like to thank the referee of the paper for his helpful suggestions.

\section{References}

[1] M. K. Aouf, Certain classes of p-valent functions with negative coefficients. II, Indian J. Pure Appl. Math. 19 (1988), no. 8, 761-767.

[2] _ A generalization of multivalent functions with negative coefficients. $I I$, Bull. Korean Math. Soc. 25 (1988), no. 2, 221-232. 
[3] _ A subclass of analytic p-valent functions with negative coefficients. I, Utilitas Math. 46 (1994), 219-231.

[4] M. K. Aouf and H. E. Darwish, Basic properties and characterizations of a certain class of analytic functions with negative coefficients. II, Utilitas Math. 46 (1994), 167-177.

[5] S. K. Lee, S. Owa, and H. M. Srivastava, Basic properties and characterizations of a certain class of analytic functions with negative coefficients, Utilitas Math. 36 (1989), 121-128.

[6] S. Owa, On a class of analytic functions with fixed second coefficient. II, Bull. Soc. Roy. Sci. Liège 53 (1984), no. 3-4, 143-157.

[7] S. Owa and M. K. Aouf, On radii of starlikeness and convexity for p-valent functions with negative coefficients, J. Fac. Sci. Tech. Kinki Univ. No, 30 (1994), 19-24.

[8] S. M. Sarargi and B. A. Uralegaddi, The radius of convexity and starlikeness for certain classes of analytic functions with negative coefficients. I, Atti Accad. Naz. Lincei Rend. Cl. Sci. Fis. Mat. Natur. (8) 65 (1978), no. 1-2, 38-42.

[9] T. Sekine and S. Owa, Note on a class of functions whose derivative has a positive real part, Bull. Soc. Roy. Sci. Liege 54 (1985), no. 4-5, 203-210.

[10] T. Yaguchi, O. Kwon, N. E. Cho, and R. Yamakawa, A generalization class of certain subclasses of p-valently analytic functions with negative coefficients, Sūrikaisekikenkyūsho Kōkyūroku No. 821 (1993), 101-111.

DEPARTMENT OF MATHEMATICS

Faculty of Science

MANSOURA UNIVERSITY

MANSOURA 35516, EGYPT

E-mail address: mkaouf1270yahoo.com 